CTP TAMU-27/93

IC/93/165

hep-th/9307043

July 1993

\title{
BRST Operator for Superconformal Algebras with Quadratic Nonlinearity
}

\author{
Z. Khviengia and E. Sezgin ${ }^{\dagger}$ \\ Center for Theoretical Physics, Texas A\&M University, \\ College Station, TX 77843-4242, USA.
}

\begin{abstract}
We construct the quantum BRST operators for a large class of superconformal and quasi-superconformal algebras with quadratic nonlinearity. The only free parameter in these algebras is the level of the (super) Kac-Moody sector. The nilpotency of the quantum BRST operator imposes a condition on the level. We find this condition for (quasi) superconformal algebras with a Kac-Moody sector based on a simple Lie algebra and for the $Z_{2} \times Z_{2}$-graded superconformal algebras with a Kac-Mody sector based on the superalgebra $\operatorname{osp}(N \mid 2 M)$ or $s \ell(N+2 \mid N)$.
\end{abstract}

$\dagger \quad$ Supported in part by the National Science Foundation, under grant PHY-9106593. 


\section{Introduction}

Superconformal algebras in two dimensions play an important role in superstring theories. With certain assumptions, such as linearity, the classification of $s o_{N}$-extended algebras was given long ago [1]. They contain a single spin 2 generator and multiplets of other generators of spin decreasing by half units down to minimum spin $2-\frac{1}{2} N$. Thus, for $N>4$ there are negative dimension generators which are problematic in any application to string theory. In fact, so far only $N=1, \ldots, 4$ superconformal algebras have found applications in string theory.

It is clearly of interest to examine the possibility of having higher extended superconformal algebras which can be used in constructing new types of superstring theories. Relaxing the requirement of linearity, higher extended superconformal algebras do actually exist. In [2] and [3], certain types of such algebras have been found. They contain the energy momentum tensor $T$, a multiplet of spin $3 / 2$ currents $G_{i}$, in the fundamental representation of $s o_{N}$ or $u_{N}$, and a multiplet of spin 1 currents $J^{a}$, in the adjoint represenation of the same algebras. A characteristic feature of these algebras is that the OPE of two spin $3 / 2$ currents contains an operator bilinear in spin 1 currents. This is very similar to the case of $W_{3}$ algebra where the OPE of two spin 3 currents contains the square of the energy-momentum tensor $[4]$.

A convenient way of characterizing the quadratically nonlinear algebras is to specify the pair $(g, \rho)$ where $g$ is the (super) Lie algebra and $\rho$ is the representation carried by the spin $3 / 2$ currents. Various possibilities for this pair have been found [5-10] in addition to those in $[2,3]$. All the known cases are listed in Tables 1-3. In particular, in the case of Table 1, as shown by Fradkin and Linetsky [5], the necessary and sufficient condition for the algebra to exist is the following identity

$$
\left(\lambda_{i j}^{a} \lambda_{k \ell}^{a}-\lambda_{j k}^{a} \lambda_{i \ell}^{a}\right)=\frac{\epsilon C_{\rho}}{d+\epsilon}\left(\eta_{i j} \eta_{k l}+\eta_{j k} \eta_{i \ell}-2 \eta_{k i} \eta_{j \ell}\right)
$$

where $\lambda_{i j}^{a}$ are te generators and $\eta_{i j}$ is the invariant tensor of the algebra $g$, and $C_{\rho}$ is the eigenvalue of the second Casimir in the $d$-dimensional representation $\rho$, and $\epsilon=-1$ for superconformal algebras, $\epsilon=1$ for quasiconformal algebras.

In the case of $N$-extended superconformal algebra for $N=3,4$, the quadratic nonlinearity can be removed by the introduction of spin $1 / 2$ and spin 0 operators [11]. Therefore we shall focus our attention to the case $N>4$. It should also be noted that the algebras $g$ can be taken to be complex. By imposing suitable reality conditions one can then obtain their real forms.

The algebras listed in Table 3 are $Z_{2} \times Z_{2}$ graded since the Kac-Moody sector itself contains bosonic and fermionic generators. In the limit of purely bosonic or fermionic KacMoody generators, these algebras reduce to some of the (quasi) superconformal algebras listed in Tables 1 and 2 . 
The difference between superconformal and quasisuperconformal algebras is that the spin $3 / 2$ currents are fermionic in the former case and bosonic in the latter case. In all these algebras the OPEs of the operators $T, G_{i}$ and $J^{a}$ are the standard ones with the exception of the OPE of $G_{i}$ with $G_{j}$ which will contain a $J J$ term. The explicit form of these OPE's will be exhibited in the following sections. Suffices to mention that these algebras admit nontrivial central extensions and that all the parameters occuring in the algebra including the central extensions are determined in terms of the level of the Kac-Moody sector of the algebra, which is the only free parameter.

An elegant general formula for the classical and quantum BRST operator for a large class of quadratically nonlinear algebras has been derived by Scoutens, Sevrin and van Nieuwenhuizen [12]. For the $s o_{N}{ }^{-}$extended superconformal algebra it was found that the existence of the BRST operator fixes the level to be $k=-2(N-3)$. Hence for the cases of interest, i.e. $N>4$, the level is negative and consequently the algebra does not admit unitary representations. In [12], it was furthemore found that a quantum BRST operator does not exist for the case of $u_{N^{-}}$extended superconformal algebra. In this paper we shall generalize these results by constructing the quantum BRST operator for all the cases listed in Table 1 (which includes the quasi-superconformal algebras) and $Z_{2} \times Z_{2}$-graded algebras with a Kac-Moody sector based on the superalgebra $\operatorname{osp}(N \mid 2 M)$ or $s \ell(N+2 \mid N)$ (see Table 3). We shall give the explicit form of the quantum BRST operators for these cases and we shall find the conditions on the levels imposed by the nilpotency of the BRST operator. For all the algebras listed in Table 1 and for the $Z_{2} \times Z_{2}$-graded algebra based on $s \ell(N+2 \mid N)$, we find that the level is still negative (eqs. (2.8a) and (3.15), respectively). In the case of $Z_{2} \times Z_{2}$-graded algebra based on $\operatorname{osp}(N \mid 2 M)$ the level can be negative or positive (eq. (3.8a)). The next two sections are devoted to the derivation of these results. Sec. 4 contains comments on the implications of these results for the existence of unitary representations and on a number of other issues.

\section{BRST Operator for (Quasi) superconformal Algebras With Bosonic Kac-Moody Sector}

The algebras of the type listed in Table 1 are generated by the energy-momentum tensor $T(z)$, the dimension $3 / 2$ supercurrents $G^{i}(z), i=1, \ldots, \operatorname{dim} \rho:=d$ and the dimension 1 currents $J^{a}(z), a=1, \ldots, \operatorname{dim} g:=D$. The operator product algebra is as follows:

$$
\begin{aligned}
T(z) T(\omega) & =\frac{\frac{1}{2} c}{(z-\omega)^{4}}+\frac{2 T(\omega)}{(z-\omega)^{2}}+\frac{\partial T(\omega)}{(z-\omega)}+\cdots, \\
T(z) G^{i}(\omega) & =\frac{\frac{3}{2} G^{i}(\omega)}{(z-\omega)^{2}}+\frac{\partial G^{i}(\omega)}{(z-\omega)}+\cdots, \\
T(z) J^{a}(\omega) & =\frac{J^{a}(\omega)}{(z-\omega)^{2}}+\frac{\partial J^{a}(\omega)}{(z-\omega)}+\cdots,
\end{aligned}
$$




$$
\begin{aligned}
G^{i}(z) G^{j}(\omega)= & \frac{b \eta^{i j}}{(z-\omega)^{3}}+\frac{\sigma \lambda_{a}^{i j} J^{a}(\omega)}{(z-\omega)^{2}}+\frac{\frac{1}{2} \sigma \lambda_{a}^{i j} \partial J^{a}(\omega)}{(z-\omega)}+\frac{2 \eta^{i j} T(\omega)}{(z-\omega)} \\
& +\frac{\gamma P_{a b}^{i j}\left(J^{a} J^{b}\right)(\omega)}{(z-\omega)}+\cdots, \\
J^{a}(z) G^{i}(\omega)= & \frac{-\lambda^{a i}{ }_{j} G^{j}(\omega)}{(z-\omega)}+\cdots, \\
J^{a}(z) J^{b}(\omega)= & \frac{-\frac{1}{2} k \psi^{2} \delta^{a b}}{(z-\omega)^{2}}+\frac{f^{a b}{ }_{c} J^{c}(\omega)}{(z-\omega)}+\cdots,
\end{aligned}
$$

where the generators $\lambda_{i j}^{a}$ and the structure constants $f_{a b}^{c}$ satisfy the relations $\dagger$

$$
\begin{array}{r}
\lambda_{a i k} \lambda_{b j}^{k}-\lambda_{b i k} \lambda_{a j}^{k}=f_{a b}{ }^{c} \lambda_{c i}{ }^{j}, \quad \\
\left(\lambda_{i j}^{a} \lambda_{k \ell}^{a}-\lambda_{j k}^{a} \lambda_{i \ell}^{a}\right)=\frac{2 \epsilon}{\sigma_{0}}\left(\eta_{i j}{ }^{j i} \eta_{k l}+\eta_{j k} \eta_{i \ell}-2 \eta_{k i} \eta_{j \ell}\right),
\end{array}
$$

and the quadratic nonlinearity is defined by $(J J)(\omega):=\frac{1}{2 \pi i} \oint d \zeta \frac{J(\zeta) J(\omega)}{(\zeta-\omega)}$. The Cartan-Killing metric $g^{a b}$ is defined as follows

$$
g_{a b}=f_{a c}^{d} f_{b d}^{c}=-C_{v} \delta_{a b}
$$

The Lie algebra is taken to be complex for the time being. The real forms and their implications for constructing unitary represantations will be briefly disussed in Sec. 4. The Dynkin index $i_{\rho}$ of the representation $\rho$ is defined by $i_{\rho}=\frac{d C_{\rho}}{D \psi^{2}}$, where $C_{\rho}$ is the eigenvalue of the second Casimir in the representation $\rho$ defined by $\lambda_{i k}^{a} \lambda_{a}^{k j}=-C_{\rho} \delta_{i}^{j}$ and $\psi^{2}$ is the square of the longest root. In a convention where the shortest root squared is 2 for all the Lie algebras, the value of $\psi^{2}$ is 2 for the simply laced Lie algebras (i.e. $A_{n}, D_{n}, E_{6}, E_{7}, E_{8}$ ), 4 for $B_{n}$, $C_{n}, F_{4}$ and 6 for $G_{2}$. The central extension in the Kac-Moody algebra is parametrized such that the unitary highest weight representations exist for positive integer values of $k$. $C_{v}$ is the eigenvalue of the second Casimir in the adjoint representation of $g$ related to the dual Coxeter number $h_{g}^{\vee}$ by $C_{v}=\psi^{2} h_{g}^{\vee}$. The raising and lowering of the indices $i, j, .$. is done by the metric $\eta_{i j}=-\epsilon \eta_{j i}$, satisfying the relation $\eta_{i k} \eta^{j k}=\delta_{i}^{j}$, by the rule: $V^{i}=\eta^{i j} V_{j}$ and $V_{i}=V^{j} \eta_{j i}$, for any quantity $V$. The parameter $\epsilon=-1$ for the superconformal algebras and $\epsilon=+1$ for the quasisuperconformal algebras. For $\epsilon=-1$ the currents $G^{i}(z)$ are fermionic, while for $\epsilon=+1$ they are bosonic.

The tensor $P_{i j}^{a b}$ is defined by

$$
P_{i j}^{a b}=\lambda_{i k}^{a} \lambda^{b k}{ }_{j}+\lambda_{i k}^{b} \lambda_{j}^{a k}+\frac{2}{\sigma_{0}} \eta_{j i} \delta^{a b}
$$

$\dagger$ We use the conventions of Ref. [5b]. It may be useful to note, however, that there are misprints in eqs. (5), (10.a,b), (11) and (14b) of Ref. [5b]). 
Note the symmetry properties: $P_{i j}^{a b}=-\epsilon P_{j i}^{a b}$ and $\lambda_{i j}^{a}=\epsilon \lambda_{j i}^{a}$. Thus $\eta^{i j} \lambda_{i j}^{a}=0$. The OPE algebra (2.1) closes provided that the parameters occuring in the algebra obey the following relations [5]

$$
\begin{aligned}
\gamma & =\frac{d(d+\epsilon)}{\psi^{4} D i_{\rho} \eta}, \quad \eta:=k+h_{g}^{\vee}+\epsilon i_{\rho}, \\
\sigma & =\frac{2 d}{\psi^{2} D i_{\rho} \eta}\left[(d+\epsilon)\left(k+h_{g}^{\vee}\right)-D i_{\rho}\right], \\
b & =\frac{k \psi^{2} \sigma}{2} \\
c & =\frac{3}{2} b+\frac{k}{\eta}(D+\epsilon d+1) .
\end{aligned}
$$

We now turn to the construction of the BRST operator corresponding to the above algebra. We introduce the pairs of ghosts $(b, c),\left(\beta^{i}, \gamma_{i}\right)$ and $\left(r^{a}, s_{a}\right)$, corresponding to the generators $T, G^{i}$ and $J^{a}$, respectively. The ghosts $\left(c, \gamma^{i}, s^{a}\right)$ have ghost number 1 and conformal dimension $\left(-1,-\frac{1}{2}, 0\right)$, respectively, while the antighosts $\left(b, \beta_{i}, r_{a}\right)$ have ghost number -1 and conformal dimension $\left(2, \frac{3}{2}, 1\right)$, respectively. They satisfy the following OPEs

$c(z) b(\omega)=\frac{1}{(z-\omega)}+\cdots, \quad \gamma^{i}(z) \beta_{j}(\omega)=\frac{\delta_{j}^{i}}{(z-\omega)}+\cdots, \quad s^{a}(z) r^{b}(\omega)=\frac{\delta^{a b}}{(z-\omega)}+\cdots$

Using the result of [12] we make an ansatz for the BRST operator depending on a number of parameters. We then verify fully the nilpotency of the BRST operator, which fixes all these parameters and in addition imposes conditions on the parameters of the algebra (2.1). For the BRST operator we find the following result:

$$
\begin{aligned}
Q= & c T+\gamma^{i} G_{i}+s^{a} J_{a}+b c \partial c+\beta_{i}\left(\frac{1}{2} \gamma^{i} \partial c-\partial \gamma^{i} c\right) \\
& -r_{a} s^{a} \partial c-b \gamma_{i} \gamma^{i}+\lambda_{a i}{ }^{j}\left(-\frac{1}{2} \epsilon \sigma_{0} r^{a} \gamma^{i} \partial \gamma_{j}+s^{a} \beta^{i} \gamma_{j}\right)-\frac{1}{2} f_{a b}{ }^{c} r_{c} s^{a} s^{b} \\
& -\frac{1}{2} \gamma P_{i j}^{a b} J_{a} r_{b} \gamma^{i} \gamma^{j}-\frac{1}{24} \gamma^{2} P_{i j}^{a b} P_{k l}^{c d} f_{a c}{ }^{e} r_{b} r_{d} r_{e} \gamma^{i} \gamma^{j} \gamma^{k} \gamma^{\ell} .
\end{aligned}
$$

Using the relations $(2.5 \mathrm{a}, \mathrm{b})$ and various group theoretical relations provided in [5], we find that the above BRST operator is nilpotent provided that the cental extensions satify the following relations:

$$
\begin{aligned}
& k=-2\left(h_{g}^{\vee}+\epsilon i_{\rho}\right) \\
& c=26+11 \epsilon d+2 D, \\
& b=16+6 \epsilon d .
\end{aligned}
$$

The first condition comes from cancellation requirement of terms bilinear in $s^{a}$. Similarly the second condition comes from the cancellation of the terms bilear in $c$, and the last condition 
from the cancellation of the terms bilinear in $\gamma^{i}$. In (2.8b) the central charge equals the sum of contributions $2(-1)^{2 s}\left(6 s^{2}-6 s+1\right)$ from each generator of conformal dimension $s$ with an aditional factor of $-\epsilon$ for spin $3 / 2$ generator. The relations $(2.8 \mathrm{~b}, \mathrm{c})$ agree with $(2.5 \mathrm{~d}, \mathrm{c})$, upon the use of (2.8a) and particular values of various group theoretical quantities listed in Table 1. The most crucial new information implied by the existence of the quantum BRST operator is the condition (2.8a) on the Kac-Moody level $k$. From Table 1 we see that $k$ will always be negative (in the case of first entry in Table 1, for $N>4$ ), which means difficulty in obtaining unitary representations of the algebra, at least in the case when its compact form is considered. In particular, we see that taking the spin $3 / 2$ currents to be bosonic does not change this conclusion. However, considering the noncompact form of the algebras listed in Table 1 may have interesting consequences for the existence of unitary representations. This point will be discussed further in Sec. 4.

We close this section with a comment on the structure of the BRST operator (2.7). The form of all the cubic in ghost terms follows from the structure constants of the linear part of the algebra. The quartic in ghost term is suggested by treating the $P J J$ term in the algebra as 'linear' in $J$ with $P J$ as a field dependent structure 'constant'. The occurence of the last term which is seventh order in ghost field, which is needed for the nilpotency of the BRST operator, can be understood from a more systematic point of view from the work of Ref. $[12]$.

\section{BRST Operator for Quadratically Nonlinear Superconformal Algebras With Super Kac-Moody Sector}

$$
\text { The Case of } \operatorname{osp}(N \mid 2 M)
$$

In this case the dimension $3 / 2$ current $G^{i}(z), i=1, \ldots, N+2 M$ carries the fundamental representation of the superalgebra $\operatorname{osp}(N \mid 2 M)$, and the spin 1 currents $J^{a}(z), a=1, \ldots, \operatorname{dim} g$ are in the adjoint representation of the same superalgebra. The first three OPE relations in (2.1) remain the same in present case, while the remaining ones read

$$
\begin{aligned}
G^{i}(z) G^{j}(\omega)= & \frac{b \eta^{j i}}{(z-\omega)^{3}}+\frac{\sigma \lambda_{a}^{i j} J^{a}(\omega)}{(z-\omega)^{2}}+\frac{\frac{1}{2} \sigma \lambda_{a}^{i j} \partial J^{a}(\omega)}{(z-\omega)}-\frac{2 \eta^{j i} T(\omega)}{(z-\omega)} \\
& +\frac{\gamma P_{a b}^{i j}\left(J^{a} J^{b}\right)(\omega)}{(z-\omega)}+\cdots \\
J^{a}(z) G^{i}(\omega)= & \frac{(-1)^{a+1} \lambda^{a i}{ }_{j} G^{j}(\omega)}{(z-\omega)}+\cdots \\
J^{a}(z) J^{b}(\omega)= & \frac{-k \eta^{b a}}{(z-\omega)^{2}}+\frac{f^{a b}{ }_{c} J^{c}(\omega)}{(z-\omega)}+\cdots .
\end{aligned}
$$

The Grassmann parity of the indices $a, i$ is indicated by the same letters and $i=1$ for $s o_{N}$, and $i=0$ for $s p_{2 M}$, while $a=0$ for the bosonic generators and $a=1$ for the fermionic 
generators. The boson-fermion parity of the currents, denoted by $n$ are: $n(T)=0, n\left(G^{i}\right)=$ $i, n\left(J^{a}\right)=a$. Thus, $G^{i}$ is fermionic for $i=1, \ldots, N$ and bosonic for $i=N+1, \ldots, N+2 M$. The metric $\eta_{a b}$ is defined in terms of the structure constans as follows

$$
f^{c d a} f_{d c}^{b}=-2\left(d_{s}-2\right) \eta^{a b}, \quad d_{s}:=N-2 M
$$

The symmetry properties of the metrics are: $\eta_{a b}=(-1)^{a b} \eta_{b a}, \eta_{i j}=(-1)^{i j+1} \eta_{j i}$. Note also that $\eta_{a c} \eta^{b c}=\delta_{a}^{b}$ and $\eta^{i k} \eta_{j k}=\delta_{i}^{j}$. The $\operatorname{osp}(N \mid 2 M)$ generators $\lambda_{i j}^{a}$ obey the algebra

$$
\lambda_{a i k} \lambda_{b j}^{k}+(-1)^{a b+1} \lambda_{b i k} \lambda_{a j}^{k}=-f_{b a c} \lambda_{i j}^{c}
$$

The structure constants are totally graded antisymmetric, e.g. $f_{a b c}=(-1)^{a b+1} f_{b a c}$. The generators satisfy the crucial identity

$$
\lambda_{i j}^{a} \lambda_{a k \ell}=-\eta_{k j} \eta_{\ell i}+(-1)^{i j+1} \eta_{k i} \eta_{\ell j}
$$

This identity agrees with (2.2) for the case of $s o_{N}$, but differs by an overall minus sign for the case of $s p_{2 M}$. The reason for this difference is that the above equation involves fermionic as well as bosonic components, and it is such that the symmetries of the left hand are maintained by the right hand side.

The tensor $P_{i j}^{a b}$ is now defined by

$$
P_{i j}^{a b}=\lambda_{i k}^{a} \lambda_{j}^{b k}+(-1)^{i j+1} \lambda_{j k}^{a} \lambda_{i}^{b k}+2 \eta^{a b} \eta_{j i}
$$

Note that $\lambda_{i j}^{a}=(-1)^{i j} \lambda_{j i}^{a}$ and the Grassmann parities obey the relation $a+i+j=0$, while $P_{i j}^{a b}=(-1)^{b a} P_{i j}^{b a}=(-1)^{i j+1} P_{j i}^{a b}$ and the Grassmann parities satisfy the relation: $a+b+i+j=0$.

The OPE algebra (2.1a-c) and (3.1) closes provided that [10]

$$
\begin{aligned}
\gamma & =\frac{1}{2\left(k+d_{s}-3\right)}, \\
\sigma & =2 \gamma\left(2 k+d_{s}-4\right), \\
b & =k \sigma, \\
c & =k \gamma\left(6 k+d_{s}^{2}-10\right) .
\end{aligned}
$$

In fact, the above equations can be obtained from those in (2.5) by evaluating them for $s o_{N}$ and then making the substitution: $d \rightarrow d_{s}$. Thus they clearly agree with the $s o_{N}$ case $(M=0)$. The comparison with the $s p_{2 M}$ case $(N=0)$ is more subtle due to the difference of normalizations in (3.4), (3.1c) and their counterparts for $s p_{2 M}$.

The construction of the quantum BRST charge proceeds as before. We introduce the ghost pairs $(b, c),\left(\beta^{i}, \gamma_{i}\right)$ and $\left(r^{a}, s_{a}\right)$ which obey the OPEs given in (2.6). We then make an 
ansatz similar to that in (2.7) and by an explicit calculation determine all the coefficients. Extreme care has to be exercised in dealing with the Grassmann parities. At the end we find the following result:

$$
\begin{aligned}
Q= & c T+\gamma^{i} G_{i}+s^{a} J_{a}+b c \partial c+\beta_{i}\left(\frac{1}{2} \gamma^{i} \partial c-\partial \gamma^{i} c\right) \\
& -r_{a} s^{a} \partial c-b \gamma^{i} \gamma_{i}-r_{a} \lambda_{i}^{a}{ }^{j} \gamma_{j} \partial \gamma^{i}+s_{a} \lambda^{a i j} \beta_{j} \gamma_{i}+\frac{1}{2} f_{b}{ }^{a c} r_{c} s_{a} s^{b} \\
& -\frac{1}{2} \gamma\left(P^{a b}\right)_{i}^{j} r_{a} J_{b} \gamma_{j} \gamma^{i}-\frac{1}{24} \gamma^{2}\left(P^{a b}\right)_{i}{ }^{j}\left(P^{c d}\right)_{k}^{\ell} f_{a e c} r^{e} r_{d} r_{b} \gamma_{j} \gamma^{i} \gamma_{\ell} \gamma^{k} .
\end{aligned}
$$

This BRST operator is nilpotent provided that the folowing relations hold:

$$
\begin{aligned}
& k=-2\left(d_{s}-3\right), \\
& c=26-12 d_{s}+d_{s}^{2}, \\
& b=16-6 d_{s} .
\end{aligned}
$$

Note that sdim $g=\frac{1}{2} N(N-1)+M(2 M+1)-2 M N=d_{s}\left(d_{s}-1\right)$, where we recall that the superdimension $d_{s}$ is defined by $d_{s}=N-2 M$. We have also used (3.2) and the relation $\lambda_{i j}^{a} \lambda^{b j i}=-2 \eta^{a b}$. The above conditions agree for the special case of $s o_{N}$ with $M=0$, while the comparison for the case of $s p_{2 M}$ is more subtle as mentioned earlier, due to the different normalizations chosen in (3.4), (3.1c) and in their counterparts for $s p_{2 M}$. Note that now the level $k$ is a positive integer for $N<2 M+3$.

The quantum BRST operator for the last case in Table 3 proceeds exactly in the same manner as described above, and its further discussion will be omitted here. Finally we discuss the $Z_{2} \times Z_{2}$ graded algebra based on $s \ell(N+2 \mid N)$.

The case of $\operatorname{s\ell }(N+2 \mid N)$

First let us discuss briefly the case of $g \ell(N \mid M)$. A real form of this algebra based on the compact superalgebra $u(N \mid M)$ was constructed in [10]. The limit $M=0$ coincides with the well known case based on $s u(N) \times u(1)[2,3]$. Interestingly enough, in [12] it was found that the quantum BRST operator does not exist for this case, because a condition that relates the Kac-Moody levels of $u(N)$ and $u(1)$ contradicts the condition implied by the closure of the OPE algebra. Given the results of the previous section, we expect that a similar situation will occur in the case of $g \ell(N \mid M)$, with $N$ of [12] being replaced by the superdimension $d_{s}=N-M$. Thus, we expect that the quantum BRST operator does not exist for the $g \ell(N \mid M)$ case. Nonetheless, an interesting situation arises for $d_{s}=2$. As was shown in [10], in that case the $g \ell(1)$ current decouples completely. Then we have $s \ell(N+2 \mid N)$ for which an invertible Cartan-Killing metric $g_{a b}$ defined as in (2.3) does exist. An interesting property of this algebra is that, despite the fact that it looks like the usual $N=4$ superconformal algebra with $S U(2)$ Kac-Moody sector, it is however different in that the quadratic nonlinearity is still there, and there are $2(N+2)$ anticommuting and $2 N$ commuting spin $3 / 2$ generators. 
Below, we shall consider this case, for which, as we shall see there does exist a quantum BRST operator.

For $s \ell(N+2 \mid N)$ case, the spin $3 / 2$ currents are $G^{i}(z)$ and $G_{i}(z), i=1, \ldots, 2 N+2$. These are independent, as there is no metric to raise and lower indices. The boson-fermion parities are $n\left(G^{i}\right)=n\left(G_{i}\right)=i$. As mentioned above there are $2(N+2)$ fermionic and $2 N$ bosonic spin $3 / 2$ generators. The nontrivial OPE's in this case are [10]

$$
\begin{aligned}
G_{i}(z) G^{j}(\omega)= & \frac{8(-1)^{i} \delta_{i}^{j}}{(z-\omega)^{3}}+\frac{2(-1)^{k} \lambda_{i}^{a}{ }^{j} J_{a}(\omega)}{(z-\omega)^{2}}+\frac{(-1)^{k} \lambda_{i}^{a j} \partial J_{a}(\omega)}{(z-\omega)}+\frac{2(-1)^{i} \delta_{i}^{j} T(\omega)}{(z-\omega)} \\
& \frac{\gamma\left(P^{a b}\right)_{i}^{j}\left(J_{a} J_{b}\right)(\omega)}{(z-\omega)}+\cdots, \\
J_{a}(z) G_{i}(\omega)= & -\frac{\lambda_{a i}{ }^{j} G_{j}(\omega)}{(z-\omega)}+\cdots, \\
J_{a}(z) G^{i}(\omega)= & \frac{(-1)^{i+i j} G^{j}(\omega) \lambda_{a j}{ }^{i}}{(z-\omega)}+\cdots, \\
J^{a}(z) J^{b}(\omega)= & \frac{-k \eta^{b a}}{(z-\omega)^{2}}+\frac{f^{a b}{ }_{c} J^{c}(\omega)}{(z-\omega)}+\cdots,
\end{aligned}
$$

where

$$
\left(P^{a b}\right)_{i}^{j}=\lambda_{i}^{a k} \lambda_{k}^{b j}+(-1)^{a b} \lambda_{i}^{b k} \lambda_{k}^{a j}+2 \delta_{i}^{j} \eta^{a b}
$$

The parameter $\gamma$ and the Virasoro central extension $c$ are related to the level $k$ as follows $[10]$

$$
\gamma=-\frac{1}{2(k+2)}, \quad c=3 k
$$

The generators are supertraceless, i.e $(-1)^{i+1} \lambda_{i}^{a i}=0$ and obey the graded commutation rule: $\lambda_{i}^{a k} \lambda_{k}^{b j}+(-1)^{a b+1} \lambda_{i}^{b k} \lambda_{k}^{a j}=-f^{b a}{ }_{c} \lambda_{i}^{c j}$. Further important identities are

$$
\begin{aligned}
& \lambda_{i}^{a j} \lambda_{a k}^{\ell}=\delta_{i}^{j} \delta_{k}^{\ell}+2(-1)^{k} \delta_{i}^{\ell} \delta_{k}^{j} \\
& f^{c d a} f_{d c}^{b}=-8 \eta^{a b}, \quad(-1)^{i+1} \lambda_{i}^{a j} \lambda_{j}^{b i}=-2 \eta^{a b} .
\end{aligned}
$$

In order to construct the BRST operator, we now introduce the ghost pairs $(b, c),\left(\beta_{i}, \gamma^{i}\right)$, $\left(\beta^{i}, \gamma_{i}\right)$ and $\left(r_{a}, s_{a}\right)$. By explicit calculation we then find the following result for the BRST operator:

$$
\begin{aligned}
Q= & c T+\gamma^{i} G_{i}+\gamma_{i} G^{i}+s^{a} J_{a}+b c \partial c+\beta_{i}\left(\frac{1}{2} \gamma^{i} \partial c-\partial \gamma^{i} c\right)+\beta^{i}\left(\frac{1}{2} \gamma_{i} \partial c-\partial \gamma_{i} c\right)+ \\
& \left.-r_{a} s^{a} \partial c+2 b \gamma_{i} \gamma^{i}+\lambda_{a i}{ }^{j} r^{a}\left(\gamma_{j} \partial \gamma^{i}-\partial \gamma_{j} \gamma^{i}\right)\right) \\
& +\lambda_{i}^{a j} s_{a}\left(\gamma_{j} \beta^{i}-\beta_{j} \gamma^{i}\right)+\frac{1}{2} f_{b}{ }^{a c} r_{c} s_{a} s^{b} \\
& +\frac{1}{4}\left(P_{a b}\right)_{i}{ }^{j} r^{a} J^{b} \gamma_{j} \gamma^{i}+\frac{1}{24}\left(P_{a b}\right)_{i}^{j}\left(P_{c d}\right)_{k}^{\ell} f_{d a e} r^{c} r_{e} r^{b} \gamma_{j} \gamma^{i} \gamma_{\ell} \gamma^{k} .
\end{aligned}
$$


The nilpotency of this operator imposes the restriction

$$
k=-4
$$

which in particular implies that $c=-12$. Unitary highest weight representations of the algebra presumably do not exist for this value of the level (see below).

\section{Comments}

So far we have considered complex Lie (super) algebras. The real forms of the algebras listed in Table 1 are well known, and the real form of the superalgebras can be found in [13]. Care must be exercised in extending these reality conditions to the full affine algebras considered here. In [5] it is argued that in the case of superconformal algebras $(\epsilon=-1)$, real forms exist with $g$ and $\rho$ real, while this is not possible in the case of quasiconformal algebras with the exception of $s \ell(N+2, C)$ giving rise to the real form $s u(N+1,1)[9]$. An interesting possibility when noncompact real forms exist is that, while the BRST condition on the level is the one that forbids unitary highest weight representations in the compact case, one may utilize a coset construction upon which one imposes $H$ invariance condition on the acceptable states, where $H$ is the maximal compact subgroup of the noncompact group. This eliminates the negative norm states, making it possible to construct unitary representations possibly with acceptable conditions on the level. This phenomenon has been studied for a number of cases [14]. (In particular, see Ref. [15] for an interesting use of $S O(d-1,2)$ Kac-Moody algebra to built a string theory in anti de Sitter space). Of course one would have to verify that the combined system of Virasoro plus Kac-Moody algebras, taking into account the quadratic nonlinearities as well, posesses a unitary represenation. If a super Sugawara construction exists, the unitarity of the Kac-Moody sector will ensure the unitarity of the whole system.

As far as the existence of the unitary highest weight representations of Kac-Moody superalgebras are concerned, this problem has been addressed in [16]. It was found that among the compact form of all such algebras only $s u(N+1 \mid 1)$ and $o s p(2 \mid 2 M)$ admit unitary highest weight representations, with a suitable condition imposed on the level $k$. They also computed the values of the Virasoro central charges for the existence of super Sugawara construction $[17,16]$. Among the compact real forms of the superalgebras considered here which may arise, only $\operatorname{su}(3 \mid 1)$ and $\operatorname{ssp}(2 \mid 2 M)$ admit unitary highest weight representations, with suitable restrictions on the level $k$ [16], which however are in conflict with the restrictions found here from the existence of the BRST operator. As to the noncompact real forms of the super Kac-Moody algebras considered here which may arise, only those based on $\operatorname{ssp}(N \mid 2, R)$ admit unitary highest weight representations, again with suitable restrictions on the level $k$ [16]. Some of those cases appear not to be in conflict with the restrictions found here. Various aspects of this problem clearly deserves further investigation. 
The problem of finding unitary representations of the quadratically nonlinear algebras with the level conditions imposed by the existence of the BRST operator is one of the important problems. A second important problem is to find a spacetime realization of superconformal field theories based on these algebras. Some realizations of these algebras are known [18], but it is not clear how they can lead to a spacetime interpretation. In these realizations, group manifolds seem naturally to arise but not Minkowskian spacetimes. It would be interesting to see if in a coset construction of the type mentioned above, or possibly in a theory based on a Wigner-Inönü type contractions of the algebras considered here, a more promising geometrical framework lending itself to some spacetime interpretation might emerge. It is certainly worthwhile to investigate possible uses of these algebras in building the internal sector of a novel string theory in the style of Gepner [19], where $N=2$ superconformal theories are used in the construction of $N=1$ supersymmetric string theories in four dimensions. It would also be interesting to see if these algebras could be used in certain statistical systems where even nonunitary representation can have physical interpretations.

Finally, it should be noted that the quadratically nonlinear algebras considered here represent a mildly nonlinear extension of the usual linear superconformal algebras. There are other, far more nonlinear versions which allow extended supersymmetry beyond $N=4$. An interesting example is the $N=8$ superconformal algebra of Ref. [20], based on a loop algebra of paralell transformations on seven spheres. In [21], the emergence of this algebra in a twistor formulation of the Green-Schwarz superstring is shown and a BRST operator is constructed. Another example is provided by the $N=8$ conformal supergravity theory of Ref. [22]. This theory has not been investigated in any detail so far.

\section{Acknowledgements}

We thank A.O. Barut, E. Bergshoeff, N. Berkovits, V. Dobrev, H. Lu, C.N. Pope and S. Schrans for helpful discussions. We also thank the Internatinal Center for Theoretical Physics, where part of this work was done, for hospitality. 


\begin{tabular}{|r|r|r|r|r|}
\hline$g$ & $\rho$ & $h_{g}^{\vee}$ & $i_{\rho}$ & References \\
\hline \multicolumn{5}{|c|}{ superconformal } \\
\hline$s O_{N}$ & $N$ & $N-2$ & 1 & {$[2,3]$} \\
\hline$s O_{7}$ & $8_{s}$ & 5 & 1 & {$[6,7]$} \\
\hline$G_{2}$ & 7 & 4 & 1 & {$[6,7]$} \\
\hline \multicolumn{5}{|c|}{ quasisuperconformal $(\epsilon=1)$} \\
\hline$s p_{2 M}$ & $2 M$ & $M+1$ & $1 / 2$ & {$[8]$} \\
\hline$s \ell_{6}$ & 20 & 6 & 3 & {$[5]$} \\
\hline$s o_{12}$ & 32 & 10 & 4 & {$[5]$} \\
\hline$E_{7}$ & 56 & 18 & 6 & {$[5]$} \\
\hline$s p_{6}$ & 14 & 4 & $5 / 2$ & {$[5]$} \\
\hline$s \ell_{2}$ & 4 & 2 & $5 / 2$ & {$[5]$} \\
\hline
\end{tabular}

Table 1. (Quasi) superconformal and algebras with simple $g$ and irreducible $\rho$.

\begin{tabular}{|c|c|c|}
\hline$g$ & $\rho$ & References \\
\hline \multicolumn{3}{|c|}{ superconformal $\quad(\epsilon=-1)$} \\
\hline$g \ell_{N}$ & $N \oplus N$ & {$[2,3]$} \\
\hline$s p_{2} \oplus s p_{2 M}$ & $(2,2 \mathrm{M})$ & {$[5,6]$} \\
\hline \multicolumn{3}{|c|}{ quasisuperconformal $(\epsilon=1)$} \\
\hline$g \ell_{N}$ & $N \oplus N$ & {$[8,9]$} \\
\hline$s p_{2} \oplus s o_{N}$ & $(2, \mathrm{~N})$ & {$[5]$} \\
\hline
\end{tabular}

Table 2. (Quasi) superconformal algebras with non-simple $g$ and/or reducible $\rho$.

\begin{tabular}{|c|c|c|}
\hline$g$ & $\rho$ & References \\
\hline$o s p(N \mid 2 M)$ & $N \mid 2 M$ & {$[10]$} \\
\hline$g \ell(N \mid M)$ & $N \mid M$ & {$[10]$} \\
\hline$s p_{2} \oplus o s p(N \mid 2 M)$ & $(2, N \mid 2 M)$ & {$[5]$} \\
\hline
\end{tabular}

Table 3. $Z_{2} \times Z_{2}$ graded superconformal algebras. 


\section{References}

1. M. Ademollo et al., Phys. Lett. B208 (1976) 447; Nucl. Phys. B111 (1976) 77.

2. V.G. Knizhnik, Theor. Math. Phys. 66 (1986) 68.

3. M. Bershadsky, Phys. Lett. B174 (1986) 285.

4. A.B. Zamolodchikov, Theor. Math. Phys. 65 (1986) 1205.

5. E.S. Fradkin and V. Y. Linetsky, Phys. Lett. B282 (1992) 352;B291 (1992) 71.

6. P. Bowcock, Nucl. Phys. B381 (1992) 415.

7. E.S. Fradkin and V.Y. Linetsky, Phys. Lett. B275 (1992) 345.

8. L.J. Romans, Nucl. Phys. B357 (1991) 549.

9. F.A. Bais, T. Tjin and P. van Driel, Nucl. Phys. B357 (1991) 632;

A.M. Polyakov, in "Physics and Mathematics of Strings" (World Scientific, 1990);

M. Bershadsky, Commun. MAth. Phys. 139 (1991) 71.

10. F. Defever, W. Troost and Z. Hasiewicz, Phys. Lett. B273 (1991) 51.

11. P. Goddard and A. Schwimmer, Phys. Lett. B214 (1988) 209.

12. K. Schoutens, A. Sevrin and P. van Nieuwenhuizen, Commun. Math. Phys. 124 (1989) 87.

13. M. Parker, J. Math. Phys. 21 (1980) 689.

14. L.J. Dixon, M.E. Peskin and J. Lykken, Nucl. Phys. B325 (1989) 329;

I. Bars, Nucl. Phys. B334 (1990) 125;

V. Dobrev and E. Sezgin, Int. J. Mod. Phys. A6 (1991) 4699.

15. E.S. Fradkin and V.Y. Linetsky, Phys. Lett. B261 (1991) 26.

16. P.D. Jarvis and R.B. Zhang, Phys. Lett. B215 (1988) 695; Nucl. Phys. B313 (1989) 205.

17. P. Goddard, D. Olive and G. Waterson, Commun. Math. Phys. 112 (1987) 591.

18. K. Schoutens, Nucl. Phys. B314 (1989) 519;

P. Matthieu, Phys. Lett. B218 (1989) 185;

K. Ito, J.O. Madsen and J.L. Petersen, preprint, NBI-HE-92-81 (October 1992).

19. D. Gepner, Nucl. Phys. B296 (1987) 757; Phys. Lett. B199 (1987) 380.

20. F. Englert, A. Sevrin, W. Troost, A. van Proeyen and P. Spindel, J. Math. Phys. 29 (1988) 281.

21. N. Berkovits, Nucl. Phys. B358 (1991) 169.

22. E. Bergshoeff, H. Nishino and E. Sezgin, Phys. Lett. B218 (1987) 167. 